\title{
Transarterial chemoembolization for hepatocellular carcinoma combined with portal vein tumor thrombosis
}

This article was published in the following Dove Press journal:

Cancer Management and Research

\author{
Wei-Fu Lv \\ Kai-Cai Liu \\ Dong Lu \\ Chun-Ze Zhou \\ De-Lei Cheng \\ Jing-Kun Xiao \\ Xing-Ming Zhang \\ Zheng-Feng Zhang
}

Department of Interventional Radiology, Anhui Provincial Hospital, The First Affiliated Hospital of University of Science and Technology of China (USTC), Hefei 23000I, Anhui Province, China
Correspondence: Wei-Fu Lv Department of Interventional Radiology, Anhui Provincial Hospital, The First Affiliated Hospital of University of Science and Technology of China (USTC), 4 Lujiang Road, Hefei 23000I, Anhui Province, China

Tel +8655162283139

Fax +8655162283139

Email wei-fulv@fsyy.ustc.edu.cn
Objectives: To explore the efficacy and influencing factors of transarterial chemoembolization (TACE) in the treatment of hepatocellular carcinoma (HCC) combined with portal vein tumor thrombosis (PVTT).

Materials and methods: The clinical data of 3,126 consecutive patients who suffered from advanced HCC and underwent TACE were retrospectively analyzed. A total of 685 patients had a combination of HCC and PVTT. Of these patients, 475 were treated with TACE (Group A) and 210 were given a supportive care (Group B). The local response and overall survival of the two groups were observed and compared, and the influencing factors were examined through Cox regression analysis.

Results: The median survival time and cumulative survival rate at 6, 12, and 24 months of Group A were higher than those of Group B $(P=0.002)$. Multiple Cox regression analysis revealed that Child-Pugh classes and PVTT grades were the independent prognostic factors affecting a patient's survival. Stratified analysis demonstrated that the survival time of patients diagnosed with grades I/II PVTT and treated with TACE was superior to that of patients provided with supportive care $(P=0.001)$, but the survival time of patients with grades III/IV PVTT with or without TACE did not significantly differ $(P=0.662)$.

Conclusion: TACE can significantly improve local response, increase cumulative survival rate, and prolong the survival duration of patients with HCC and grades I/II PVTT, whereas the efficacy of TACE for patients with grades III/IV PVTT should be further verified, although their local responses were improved. Child-Pugh classes and PVTT grades are essential factors influencing patient prognosis.

Keywords: HCC, TACE, portal vein tumor thrombus, survival analysis

\section{Introduction}

Hepatocellular carcinoma (HCC) is the fifth most common cancer in the world. The tumor tends to invade the portal vein, thereby forming portal vein tumor thrombosis (PVTT). The incidence of PVTT in patients with HCC is $44.0 \%-62.2 \%{ }^{1}$ PVTT can induce intrahepatic dissemination and early recurrence after radical resection. Moreover, bleeding in the esophagus and the stomach fundus because of portal hypertension frequently occurs in patients with HCC and PVTT. Therefore, the overall prognosis of patients with HCC and PVTT is poor, and their median survival time is 2.7 months if they are not given the optimal treatment. ${ }^{2}$

According to the widely accepted Barcelona Clinic Liver Cancer (BCLC) staging protocol, ${ }^{3,4} \mathrm{HCC}$ combined with PVTT is classified as stage $\mathrm{C}$, and its only 
recommended treatment option is palliative therapy with sorafenib care. ${ }^{5-9}$ In the Sorafenib HCC Assessment Randomized Protocol (SHARP) trial, which is a large Phase III, multicenter, randomized, double-blind, placebo-controlled trial, sorafenib treatment is compared with placebo among 602 patients with Child-Pugh class A liver function, and its results show a significant difference in overall survival (OS; 10.7 vs 7.9 months, $P<0.001) .{ }^{6}$ And, in the subgroup analysis, patients with macroscopic vascular invasion in the sorafenib group and in the control group have OS of 8.1 and 4.9 months, respectively. ${ }^{8}$ However, Jeong et al ${ }^{10}$ reported that patients with HCC and grades III/IV PVTT treated with sorafenib therapy had an OS of 3.1 months, which is an unsatisfactory outcome. Besides, some patients may experience severe adverse reactions to sorafenib; as such, reducing doses or discontinuing treatment should be given. ${ }^{11,12}$ Obviously, additional management strategies need to be identified and optimized to improve the treatment benefits in clinical practice.

Transarterial chemoembolization (TACE) is an essential method for patient with HCC combined with PVTT in many medical centers. ${ }^{12-17}$ However, the efficacy and safety of TACE remain controversial or unconfirmed.

Patients with HCC associated with PVTT are exposed to a broad spectrum of hemodynamic changes because of the different extents of portal vein invasion, and these patients may encounter various tumor biological characteristics, liver function scores, and physical performance statuses. As such, previous studies that reported the efficacy of TACE from different medical centers yielded controversial results. ${ }^{14-16,18}$ Consequently, further studies should aim to investigate the efficacy and risk factors of TACE through stratified analysis with large-scale samples.

In the present study, 475 consecutive patients with HCC combined with PVTT were treated with TACE, and 210 patients were subjected to supportive care in the same period. The clinical data were retrospectively analyzed. PVTT grades and liver function classes were examined through stratified analysis. The potential risk factors influencing a patient's survival were assessed to evaluate the efficiency and influencing factors of TACE for patients with HCC associated with PVTT.

\section{Materials and methods}

\section{Patients}

This study was approved by our institutional ethics committee, and all the patients provided their written informed consent. We searched our institution's electronic database from January 2009 to December 2015 and identified 3,126 consecutive patients who suffered from HCC. Among them, 685 patients were diagnosed with HCC combined with PVTT, including 589 men and 96 women, with an average age of 54.9 years (range, 31-79 years). Of these patients, 475 underwent TACE (Group A), and 210 accepted conservative supportive care (Group B). In Group A, the patients received TACE based on the following criteria: 1) Child-Pugh class A or B, 2) an Eastern Cooperative Oncology Group performance status score from 0 to 2,3 ) tumors size $<70 \%$ of the whole liver volume, and 4) no serious concurrent medical illness, and 5) age $\geq 18$ years. The exclusion criteria were as follows: 1) patients with massive ascites, 2) patients with hepatic encephalopathy, and 3 ) patients with serious diseases in other visceral organs. The related clinical data of the patients were collected, and no statistical differences were found in the baseline characteristics between the two groups (Table 1).

\section{Diagnosis and definitions}

HCC was diagnosed by conducting a histology examination or by the criteria endorsed by the American Association for the Study of Liver Diseases. ${ }^{3}$ The classification of PVTT was determined through enhanced computed tomography (CT) or MRI examinations in accordance with the classification recommendations proposed by the Liver Cancer Study Group of Japan ${ }^{19}$ : grade I, tumor thrombus involving the segmental branches of the portal vein or above; grade II, tumor thrombus involving the right/left portal vein; grade III, tumor thrombus involving the main portal vein; and grade IV, tumor thrombus involving the superior mesenteric vein or the splenic vein.

\section{Treatment methods \\ Group A}

The modified Seldinger method was used to puncture the right femoral artery, and a $5.0 \mathrm{Fr}(1 \mathrm{Fr}=0.333 \mathrm{~mm})$ micropuncture introducer set (Terumo, Tokyo, Japan) was utilized to access the femoral artery. A 4.0 or 5.0 Fr RH catheter (Cook, Bloomington, IN, USA) was introduced into the abdominal aorta and then into the celiac trunk. Hepatic arterial angiographies and indirect portography were performed. A 2.8 Fr microcatheter (Progreat ${ }^{\mathrm{TM}}$, Terumo, Tokyo, Japan) was inserted to the arteries feeding the tumor. An emulsion of 5-20 mL of lipiodol (Andre Guerbet, Aulnay-sous-Bois, France) and epirubicin (30-60 mg) was mixed and infused into the feeding arteries under fluoroscopic monitoring. When lipiodol infusion was finished, oxaliplatin (100-200 mg) or fluorouracil glycosides (500-1,000 mg) were infused through the catheter, and polyvinyl alcohol particles with diameters of $75-150 \mu \mathrm{m}$ were used to embolize the tumor vessels. 
Table I The baseline characteristics of patients and their comparison between Group A and Group B

\begin{tabular}{|c|c|c|c|}
\hline Variables & $\begin{array}{l}\text { Group A } \\
(n=475)\end{array}$ & $\begin{array}{l}\text { Group B } \\
(n=2 \mid 0)\end{array}$ & $P$-value \\
\hline Age (years) & $54.60 \pm 11.37$ & $55.25 \pm|| .4 \mid$ & 0.626 \\
\hline Gender (\%) & & & 0.667 \\
\hline Female & $64(13.5)$ & $32(15.2)$ & \\
\hline Male & $4 I I(86.5)$ & I $78(84.8)$ & \\
\hline HBV (\%) & & & 0.583 \\
\hline No & $164(34.6)$ & 67 (31.9) & \\
\hline Yes & $311(65.4)$ & $143(68.1)$ & \\
\hline Cirrhosis (\%) & & & 0.996 \\
\hline No & $221(46.5)$ & $113(53.8)$ & \\
\hline Yes & $254(53.5)$ & $97(46.2)$ & \\
\hline Ascites (\%) & & & 0.352 \\
\hline No & 340 (7I.6) & I 62 (77.I) & \\
\hline Yes & $135(28.4)$ & $48(22.9)$ & \\
\hline Child-Pugh class (\%) & & & 0.464 \\
\hline A & $269(56.6)$ & $128(6 \mid .0)$ & \\
\hline B & $206(43.4)$ & $82(39.0)$ & \\
\hline ECOG scores (\%) & & & 0.469 \\
\hline $0-1$ & 291 (6I.3) & $120(57.1)$ & \\
\hline 2 & $184(38.7)$ & $90(42.9)$ & \\
\hline AFP $(\mathrm{ng} / \mathrm{mL})$ & $27557 \pm 789.91$ & $26283 \pm 835.27$ & 0.895 \\
\hline Tumor number (\%) & & & 0.835 \\
\hline Single & $299(62.9)$ & $134(63.8)$ & \\
\hline Multiple & $176(37.1)$ & $76(36.2)$ & \\
\hline Tumor size $(\mathrm{cm})$ & & & 0.095 \\
\hline$\leq 5$ & $12(2.5)$ & $8(3.8)$ & \\
\hline $5-10$ & $199(41.9)$ & $107(51.0)$ & \\
\hline$\geq 10$ & $264(55.6)$ & $95(45.2)$ & \\
\hline PVTT grade (\%) & & & 0.617 \\
\hline Grade I/II & $360(75.8)$ & $165(78.6)$ & \\
\hline Grade III/IV & $115(24.2)$ & $45(2 \mid .4)$ & \\
\hline Metastasis (\%) & & & 0.678 \\
\hline No & $346(72.8)$ & I50 (7I.4) & \\
\hline Yes & $129(27.2)$ & $60(28.6)$ & \\
\hline Antiviral therapy (\%) & & & 0.686 \\
\hline No & $302(63.6)$ & $138(65.7)$ & \\
\hline Yes & $173(36.4)$ & $72(34.3)$ & \\
\hline
\end{tabular}

Abbreviations: AFP, alpha-fetoprotein; ECOG, Eastern Cooperative Oncology Group; HBV, hepatitis B virus; PVTT, portal vein tumor thrombosis.

\section{Group B}

TACE was not conducted in this group; instead, liver function protection, nutrition support care, and related symptomatic, sorafenib, or systemic treatments were given. Of the 210 patients, $35(16.67 \%)$ were given Chinese traditional medicine, 23 (10.95\%) were treated with sorafenib, and 17 $(8.10 \%)$ were subjected to chemotherapy (oxaliplatin combined with 5-fluorouracil or doxorubicin).

\section{Efficacy evaluation and follow-up}

Patients were followed-up every 6-8 weeks after treatment. Their blood routine, liver function, bleeding and coagulation time, and alpha-fetoprotein were re-examined, and upper abdominal enhanced CT or MRI was conducted. The treatment strategies for another time were determined in accordance with the treatment response. Tumor response was recorded in every follow-up and classified after 6 months of treatment in accordance with the modified Response Evaluation Criteria in Solid Tumors for HCC, ${ }^{20-22}$ as complete response $(\mathrm{CR})$, partial response (PR), stable disease (SD), or progressive disease (PD). The objective response rate $(\mathrm{ORR})$ was defined as $(\mathrm{CR}+\mathrm{PR}) /$ total cases $\times 100 \%$, and disease control rate (DCR) was defined as $(\mathrm{CR}+\mathrm{PR}+\mathrm{SD}) /$ total cases $\times 100 \%$. The study endpoint was OS, which was defined as the time from the start of treatment until death from any cause or the last medical examination. All of the patients were followed-up until death or until December 31, 2016.

\section{Statistical analysis}

Data were analyzed with SPSS 17.0 (SPSS Inc., Chicago, IL, USA). Measurement data were expressed as mean \pm standard deviation, and two groups were compared through an independent sample $t$-test. Enumeration data were analyzed with a chi-squared test. Probable influencing prognostic factors were screened through Cox univariate and multiple regression analyses. The OS time of the two groups was compared by using a Kaplan-Meier estimator. $P<0.05$ was considered to indicate a significant difference.

\section{Results}

\section{Near-term local response}

The efficacy of the treatment in the two groups was initially evaluated 6-8 weeks (median, 7.1 weeks) after it was administered. A total of 28 patients died until the first follow-up, that is, 5 in Group A and 23 in Group B. The difference in the number of deaths was significant between Group A and Group B $\left(\chi^{2}=35.949, P<0.001\right)$. Of the 475 patients in Group A, 8 CR, 122 PR, 168 SD, and 177 PD were recorded. Of the 210 patients in Group B, 0 CR, 0 PR, 65 SD, and 145 PD were documented. The DCR and ORR of Group A were significantly higher than those of Group $\mathrm{B}\left(\chi^{2}=64.482, P<0.001\right.$; $\left.\chi^{2}=44.396, P<0.001\right)$.

\section{Overall survival}

Until the end of follow-up, 78 patients survived, 25 were lost to follow-up, and 582 died (ie, 403 patients in Group A and 179 patients in Group B). The median survival time of the patients enrolled in the present study was 6.37 months (1.3-58.6 months). The median survival times of the patients in Group A and Group B were 7.49 and 4.90 months, respectively. The cumulative survival rates of Group A at 6, 12, and 
24 months were $57.3 \%, 27.3 \%$, and $12.1 \%$, respectively, and those of Group B were $37.9 \%, 12.2 \%$, and 3.1\%, respectively. These values significantly differed ( $P=0.002$; Figure 1$)$.

\section{Prognostic factors}

In the present study, thirteen factors potentially influencing patient's survival were included (Table 2). Univariate analysis showed that tumor size, Child-Pugh class, and PVTT grade might affect the patient's survival. Multiple regression analysis revealed that Child-Pugh class and PVTT grade were the independent risk factors influencing the survival of patients with HCC and PVTT.

\section{TACE efficacy in different grades of PVTT}

Stratified analysis was conducted to further investigate the differences in the TACE efficacy in patients with different PVTT grades and liver function classes. The results showed that the median survival times of patients with grades I/II PVTT in Group A and Group B were 8.42 and 5.22 months, respectively. Furthermore, the cumulative survival rates of Group A at 6, 12, and 24 months were $62.7 \%, 30.8 \%$, and $14.8 \%$, respectively, and those of Group B were $41.3 \%$, $11.5 \%$, and $2.3 \%$, respectively. The median survival time and cumulative survival rate of Group A with grades I/II PVTT were significantly higher than those of Group B $(P=0.001$; Figure 2). Conversely, no differences in median survival time ( 5.03 vs 4.06 months) and the cumulative survival rates $(40.5 \%, 16.6 \%$, and $4.3 \%$ vs $26.1 \%, 13.0 \%$, and $4.5 \%$ at 6 , 12 , and 24 months) were found between Group A and Group $\mathrm{B}$ in patients with grades III/IV PVTT ( $P=0.662$; Figure 3$)$.

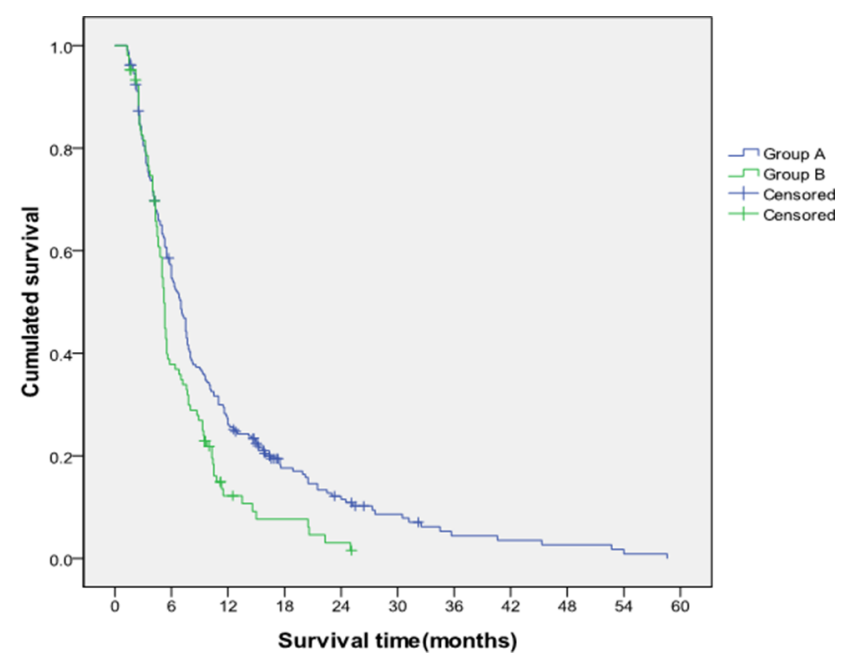

Figure I Comparison of cumulative survival between Group A and Group B. Notes: The cumulative survival rates of Group $A$ at 6, 12, and 24 months were significantly higher than those of Group B, and the survival time of Group A was longer than that of Group B $(P=0.002)$.

\section{TACE efficacy in different classes of liver function}

The median survival times of patients with Child-Pugh class A of liver function in Group A and Group B were 8.79 and 5.44 months, respectively. Furthermore, the cumulative survival rates at 6,12 , and 24 months were $63.4 \%, 34.3 \%$, and $17.0 \%$ in Group A and $44.5 \%, 12.5 \%$, and $2.5 \%$ in Group B, respectively, and these values significantly differed $(P=0.003$; Figure 4). No differences in the median survival time (5.91 vs 4.23 months) and cumulative survival rates $(49.0 \%, 17.7 \%$, and $6.2 \%$ vs $26.9 \%, 10.8 \%$, and $3.6 \%$ ) at 6,12 , and 24 months were found in patients with Child-Pugh class B in Group A and Group B $(P=0.180)$.

\section{Discussion}

The treatment strategy of patients with HCC combined with PVTT remains controversial. Previous studies showed that TACE for patients with HCC combined with PVTT can extend their survival time. ${ }^{15,16,21}$ In a series of prospective randomized controlled trials by Luo et al, ${ }^{16} 164$ patients with HCC and PVTT were divided into two groups, namely, TACE group with 84 patients and conservative treatment group with 80 patients. The median survival times of the TACE group and the conservative treatment group were 7.1 and 4.1 months, respectively. The 1- and 2-year survival rates of the former were higher than those of the latter. The safety and effectiveness of TACE were thus confirmed. Similarly, Uraki et $\mathrm{al}^{23}$ retrospectively analyzed 61 patients with HCC combined with PVTT. After patients undergo TACE, tumors with a volume reduction of $\geq 25 \%$ account for $43 \%$, and the median survival times of TACE and supportive treatment groups are 7.49 and 4.90 months, respectively. ${ }^{23}$ Their 1-, 3-, and 5-year survival rates are $42 \%, 11 \%$, and $3 \%$, respectively. ${ }^{23}$ Consistent with previous results, our findings further confirmed that TACE could significantly prolong a patient's survival time and be administered to patients with HCC combined with PVTT. We speculated that TACE can block feeding arteries, thereby controlling tumor and PVTT progression because the blood supply of HCC lesions and tumor thrombus mostly originates from the hepatic artery. ${ }^{24}$ Subsequently, the formation of collateral circulation or the recanalization of portal vein can reduce the risk of hepatic ischemia. ${ }^{25}$

In the present study, the median survival time of TACE (7.49 months) was similar to the result of the SHARP trial (8.1 months) in patients with HCC and vascular invasion. TACE cannot prolong the survival time compared with that in the SHARP trial. ${ }^{8}$ However, in the SHARP trial, 95\% of patients are categorized as Child-Pugh class A liver 
Table 2 The univariate analysis of prognostic factors in HCC patients with PVTT

\begin{tabular}{|c|c|c|c|c|}
\hline Variables & Number (\%) & Risk ratio & $P$-value & $95 \% \mathrm{Cl}$ \\
\hline Age (years) & & 0.954 & 0.688 & $0.758-1.200$ \\
\hline$\leq 50$ & $260(37.9)$ & & & \\
\hline$>50$ & $425(62.1)$ & & & \\
\hline Gender & & 0.719 & 0.053 & $0.515-1.004$ \\
\hline Female & $96(14.0)$ & & & \\
\hline Male & $589(86.0)$ & & & \\
\hline HBV(+) & & 0.848 & 0.178 & $0.667-1.078$ \\
\hline No & $231(33.7)$ & & & \\
\hline Yes & $454(66.3)$ & & & \\
\hline Cirrhosis & & 0.807 & 0.063 & $0.644-1.012$ \\
\hline No & $334(48.8)$ & & & \\
\hline Yes & $35 I(5 I .2)$ & & & \\
\hline Ascites & & 1.171 & 0.221 & $0.909-1.508$ \\
\hline No & $502(73.3)$ & & & \\
\hline Yes & $183(26.7)$ & & & \\
\hline Child-Pugh class & & 1.433 & 0.002 & $1.141-1.800$ \\
\hline A & 397 (57.9) & & & \\
\hline B & $288(42.1)$ & & & \\
\hline ECOG scores & & 1.055 & 0.647 & $0.840-1.325$ \\
\hline $0-1$ & $4 I I(60.0)$ & & & \\
\hline 2 & $274(40.0)$ & & & \\
\hline AFP (ng/mL) & & 1.096 & 0.463 & $0.858-1.400$ \\
\hline$\leq 200$ & $215(31.4)$ & & & \\
\hline$>200$ & $470(68.6)$ & & & \\
\hline Tumor number & & 0.784 & 0.340 & $0.455-1.351$ \\
\hline Single & $433(63.2)$ & & & \\
\hline Multiple & $252(36.8)$ & & & \\
\hline Tumor size (cm) & & & 0.008 & \\
\hline$\leq 5$ & $20(2.9)$ & & & \\
\hline $5-10$ & $306(44.7)$ & 1.009 & $0.97 I^{a}$ & $0.621-1.640$ \\
\hline$\geq 10$ & $359(52.4)$ & 1.443 & $0.130^{\mathrm{b}}$ & $0.897-2.320$ \\
\hline PVTT grade & & 1.428 & 0.008 & $1.098-1.857$ \\
\hline $\mathrm{I} / \mathrm{II}$ & $525(76.6)$ & & & \\
\hline III/IV & $160(23.4)$ & & & \\
\hline Metastasis & & 0.807 & 0.101 & $0.624-1.043$ \\
\hline No & $496(72.4)$ & & & \\
\hline Yes & $189(27.6)$ & & & \\
\hline Antiviral therapy & & 0.860 & 0.208 & $0.680-1.087$ \\
\hline No & $245(35.8)$ & & & \\
\hline Yes & $439(64.2)$ & & & \\
\hline
\end{tabular}

Notes: a Comparison of prognostic factor for $\leq 5 \mathrm{~cm}$ tumor size with that of $5-10 \mathrm{~cm}$ size. ${ }^{b}$ Comparison of prognostic factor for $\leq 5 \mathrm{~cm}$ tumor size with that of $\geq 10 \mathrm{~cm}$ size. Abbreviations: AFP, alpha-fetoprotein; ECOG, Eastern Cooperative Oncology Group; HBV, hepatitis B virus; HCC, hepatocellular carcinoma; PVTT, portal vein tumor thrombosis.

function and tumor thrombosis is limited as macroscopic vascular invasion. ${ }^{8}$ In the present study, only $56.6 \%$ of the patients had Child-Pugh class A liver function and $24.2 \%$ of the patients had tumor thrombosis extending to the main portal vein, the splenic vein, or the superior mesenteric vein (grades III/IV PVTT). Previous studies confirmed that the classification of liver function and the extent of PVTT invasion are the significant influencing factors of sorafenib efficacy for patients with PVTT. Several studies have revealed that patients with Child-Pugh B/C have a short OS and an increased incidence of severe adverse events. ${ }^{12-15}$ Song et $\mathrm{al}^{26}$ reported that sorafenib therapy for patients with $\mathrm{HCC}$ and grades II-IV PVTT yields a response rate of $13.3 \%$, a disease control rate of $44 \%$, a median time to progression of 2.1 months, and a median survival time of 5.5 months. Overall, TACE can be cautiously applied to patients who suffer from HCC combined with PVTT but do not have opportunities or indications to use sorafenib.

This study showed that the liver function classification was an independent factor influencing the survival of 


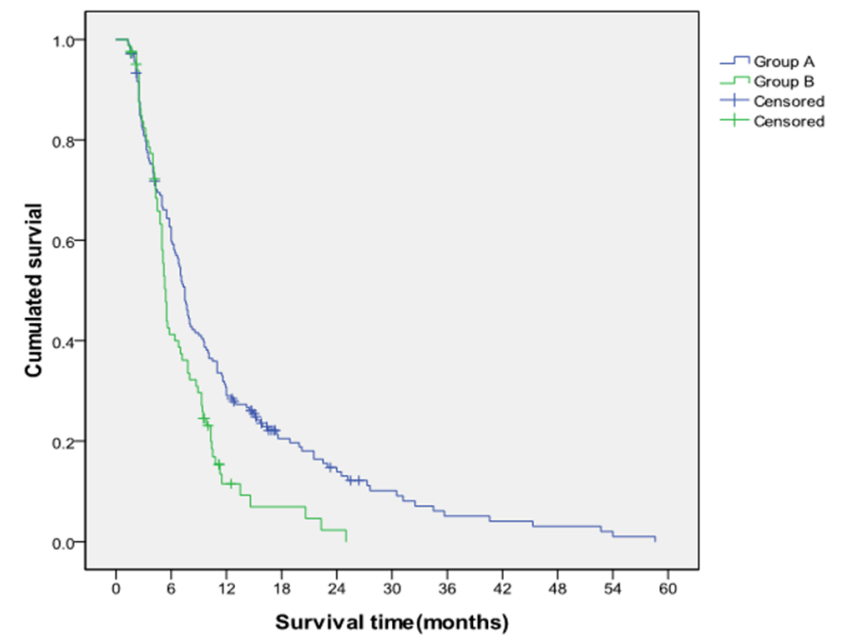

Figure 2 Comparison of cumulative survival between Group A and Group B for patients with grades I/II PVTT.

Notes: The cumulative survival rates of Group A at 6, 12, and 24 months were significantly higher than those of Group B, and the survival time of Group A was longer than that of Group B $(P=0.001)$.

Abbreviation: PVTT, portal vein tumor thrombosis.

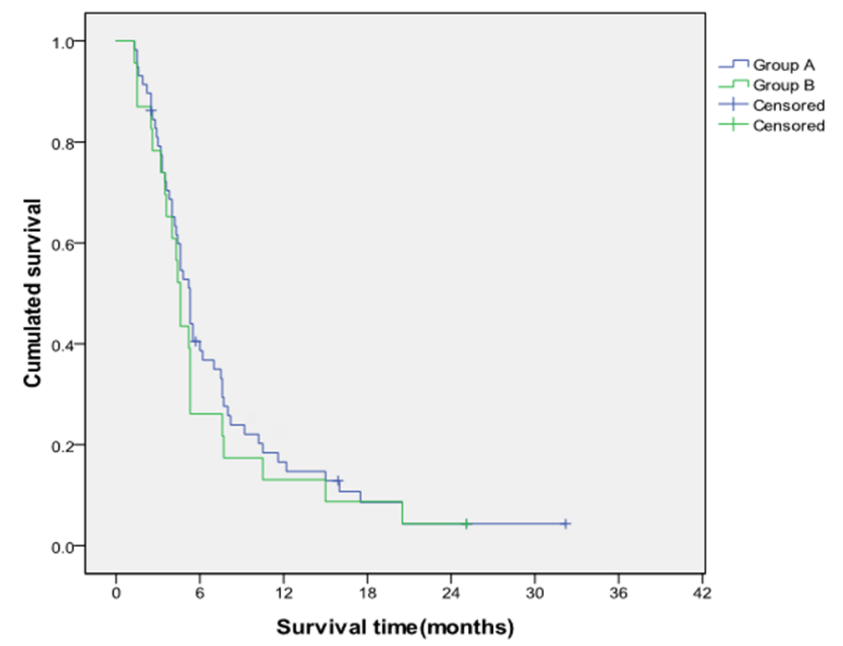

Figure 3 Comparison of cumulative survival between Group A and Group B for patients with grades III/IV PVTT.

Note: No significant difference was observed in the cumulative survival between Group A and Group B ( $P=0.662$ ).

Abbreviation: PVTT, portal vein tumor thrombosis.

patients with HCC and PVTT. TACE might aggravate liver injury because patients had a limited liver function, while Child-Pugh classification could help elucidate the compensatory capacity of a patient's liver function. Georgiades et al ${ }^{14}$ indicated that Child-Pugh classification is the most closely related prognostic factor of HCC combined with PVTT, and the median survival times of patients with Child-Pugh classes $A$ and $B$ are 11 and 4 months, respectively. The present study suggested that the median survival times of the patients with Child-Pugh class A in Group A and Group B were 8.79 and

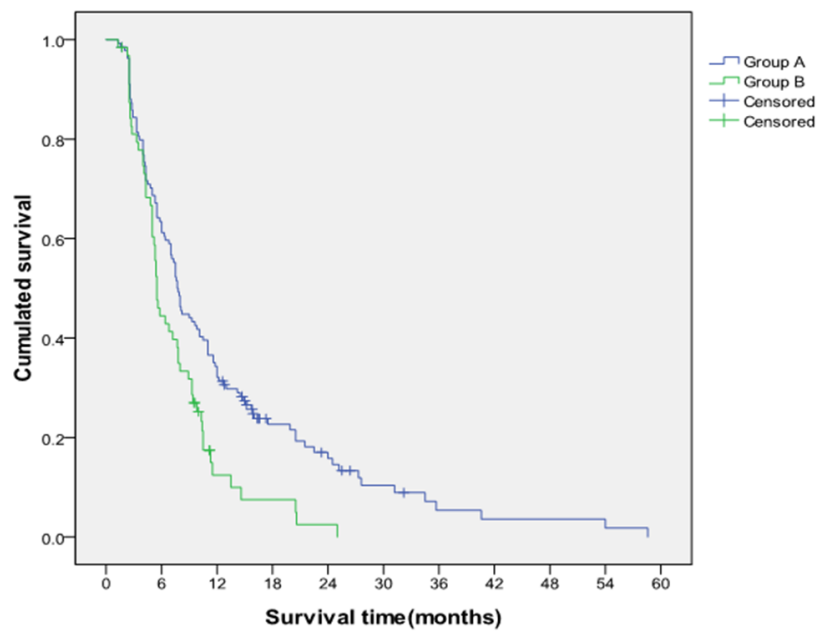

Figure 4 Comparison of cumulative survival between Group A and Group B for patients with Child-Pugh class $A$ of liver function.

Notes: The cumulative survival rates of Group A at 6, 12, and 24 months were significantly higher than those of Group B, and the median survival time of Group $A$ was significantly longer than that of Group $B(P=0.003)$.

5.44 months, respectively. The cumulative survival rates at 6 , 12 , and 24 months were $63.4 \%, 34.3 \%$, and $17.0 \%$ in Group A and $44.5 \%, 12.5 \%$, and $2.5 \%$ in Group B, respectively. A significant difference was observed in the two groups $(P=0.003)$. By contrast, no differences were found in the survival time (5.91 vs 4.23 months) and the cumulative survival rates $(49.0 \%, 17.7 \%$, and $6.2 \%$ vs $26.9 \%, 10.8 \%$, and $3.6 \%)$ at 6, 12, and 24 months between Group A and Group $\mathrm{B}$ in patients with Child-Pugh class $\mathrm{B}(P=0.180)$. Obviously, patients with Child-Pugh class A could benefit from TACE. These results revealed that TACE is the indication for patients with HCC combined with PVTT.

PVTT grade is also an independent risk factor influencing the survival of patients with HCC and PVTT. Some studies have indicated that TACE is unsuitable for patients with HCC combined with PVTT, ${ }^{4,14}$ but other studies have proposed that TACE is safe and feasible for these patients. ${ }^{13,27}$ Niu et $\mathrm{a}^{27}$ performed a prospective study to compare the survival time of patients with HCC and PVTT in TACE and control groups and found that the survival time of the two groups differs; furthermore, the effect of TACE is better than that of the control group. Kim et $\mathrm{al}^{13}$ analyzed the treatment benefit of patients diagnosed with HCC combined with PVTT and subjected to TACE. They found that the median survival times of the patients with HCC combined with grades III and IV PVTT are 7.0 and 3.9 months, respectively, and their 2-year cumulative survival rates are $27 \%$ and $11.6 \%$, respectively $(P<0.001)$. We speculated that the formation of PVTT could cause portal hypertension, liver function deterioration, 
extensive intrahepatic metastasis, and ascites, thereby affecting treatment efficacy and prognosis.

In the present study, PVTT was further divided into two groups based on the invasion of the main portal trunk. The stratified analysis revealed that the median survival times of the patients with grades I/II PVTT in Group A and Group B were 8.42 and 5.22 months, respectively, and their cumulative survival rates at 6,12 , and 24 months were $62.7 \%$, $30.8 \%$, and $14.8 \%$ in Group A and $41.3 \%, 11.5 \%$, and $2.3 \%$ in Group $\mathrm{B}$, respectively $(P=0.001)$. The median survival times of Group A and Group B with grades III/IV PVTT were 5.03 and 4.06 months, respectively. The cumulative survival rates at 6,12 , and 24 months were $40.5 \%, 16.6 \%$, and $4.3 \%$ in Group A and $26.1 \%, 13.0 \%$, and $4.5 \%$ in Group $\mathrm{B}$, respectively. No significant differences were found in the survival time of patients with grades III/IV PVTT between Group A and Group B $(P=0.662)$. TACE helped prolong the survival time of patients with grades I/II PVTT, but no significant differences were found in the extension of the survival time of patients with grades III/IV PVTT between Group A and Group B. By comparison, Niu et $\mathrm{al}^{27}$ showed that the median survival times of patients with $\mathrm{HCC}$ and grades I, II, III, and IV PVTT are 19, 11, 7.1, and 4 months in the TACE group and 4, 1.4, 1.3, and 1.0 months in the control group, respectively. These findings implied that the survival times of patients with grades I, II, III, and IV PVTT differed between the two groups.

Several limitations of this study should be noted. This study was retrospective, and the included samples were not random, thereby leading to selection bias in determining the treatment regimen, although the baseline characteristics were similar between the two groups, suggesting that the degree of bias might not have been large. A prospective randomized study should be conducted to evaluate the benefits of TACE among patients with HCC and PVTT. Second, the treatment regimens in the control group of this study were ununified, possibly affecting the OS. Third, the diagnosis and grades of PVTT were determined through enhanced CT scans or MRI without pathologic confirmation. Thrombogenesis tends to occur on the basis of PVTT, which can broaden the extent of portal vein occlusion, thereby influencing the accuracy of PVTT grading because accurate imaging modalities for PVTT grading could not be obtained.

\section{Conclusion}

TACE might have some limited efficacies for patients with HCC combined with PVTT. This treatment could significantly improve local response, increase cumulative survival rate, and prolong the survival duration of patients with HCC and grades I/II PVTT, although the efficacy of TACE for patients with grades III/IV PVTT should be further verified. Child-Pugh classes and PVTT grades were essential factors influencing patient prognosis.

\section{Ethical approval}

This study was approved by the Ethics Committee of Anhui Provincial Hospital in China and abided with the Helsinki Declaration.

\section{Acknowledgment}

This study was supported by the Anhui project for tackle hard-nut problems in science and technology (grant number: 1704a0802152) and the Anhui Provincial Natural Science Foundation (grant number: 1808085MH254).

\section{Author contributions}

W-FL conceived, designed, and revised the manuscript. K-CL, DL, and C-ZZ drafted the manuscript. D-LC, J-KX, $\mathrm{X}-\mathrm{MZ}$, and Z-FZ acquired the data and performed data analysis. All authors contributed toward data analysis, drafting and revising the paper and agree to be accountable for all aspects of the work. All the authors read and approved the final manuscript.

\section{Disclosure}

The authors report no conflicts of interest in this work.

\section{References}

1. Takizawa D, Kakizaki S, Sohara N, et al. Hepatocellular carcinoma with portal vein tumor thrombosis: clinical characteristics, prognosis, and patient survival analysis. Dig Dis Sci. 2007;52(11):3290-3295.

2. Llovet JM, Bustamante J, Castells A, et al. Natural history of untreated nonsurgical hepatocellular carcinoma: rationale for the design and evaluation of therapeutic trials. Hepatology. 1999;29(1):62-67.

3. Bruix J, Sherman M. American Association for the Study of Liver Diseases. Management of hepatocellular carcinoma: an update. Hepatology. 2011;53(3):1020-1022.

4. Villanueva A, Hernandez-Gea V, Llovet JM. Medical therapies for hepatocellular carcinoma: a critical view of the evidence. Nat Rev Gastroenterol Hepatol. 2013;10(1):34-42.

5. Llovet JM, Fuster J, Bruix J; Barcelona-Clínic Liver Cancer Group. The Barcelona approach: diagnosis, staging, and treatment of hepatocellular carcinoma. Liver Transpl. 2004;10(2 Suppl 1):S115-S120.

6. Llovet JM, Ricci S, Mazzaferro V, et al. Sorafenib in advanced hepatocellular carcinoma. $N$ Engl J Med. 2008;359(4):378-390.

7. Fu QH, Zhang Q, Bai XL, et al. Sorafenib enhances effects of transarterial chemoembolization for hepatocellular carcinoma: a systematic review and meta-analysis. J Cancer Res Clin Oncol. 2014;140(8):1429-1440.

8. Bruix J, Raoul JL, Sherman M, et al. Efficacy and safety of sorafenib in patients with advanced hepatocellular carcinoma: subanalyses of a phase III trial. J Hepatol. 2012;57(4):821-829. 
9. Welker MW, Trojan J. Antiangiogenic treatment in hepatocellular carcinoma: the balance of efficacy and safety. Cancer Manag Res. 2013;5:337-347.

10. Jeong SW, Jang JY, Shim KY, et al. Practical effect of sorafenib monotherapy on advanced hepatocellular carcinoma and portal vein tumor thrombosis. Gut Liver. 2013;7(6):696-703.

11. Yau T, Chan P, Ng KK, et al. Phase 2 open-label study of single-agent sorafenib in treating advanced hepatocellular carcinoma in a hepatitis B-endemic Asian population: presence of lung metastasis predicts poor response. Cancer. 2009;115(2):428-436.

12. Nojiri S, Kusakabe A, Fujiwara K, et al. Clinical factors related to long-term administration of sorafenib in patients with hepatocellular carcinoma. Cancer Manag Res. 2012;4:423-429.

13. Kim KM, Kim JH, Park IS, et al. Reappraisal of repeated transarterial chemoembolization in the treatment of hepatocellular carcinoma with portal vein invasion. J Gastroenterol Hepatol. 2009;24(5):806-814.

14. Georgiades CS, Hong K, D’Angelo M, Geschwind JF. Safety and efficacy of transarterial chemoembolization in patients with unresectable hepatocellular carcinoma and portal vein thrombosis. J Vasc Interv Radiol. 2005;16(12):1653-1659.

15. Lee HS, Kim JS, Choi IJ, Chung JW, Park JH, Kim CY. The safety and efficacy of transcatheter arterial chemoembolization in the treatment of patients with hepatocellular carcinoma and main portal vein obstruction. A prospective controlled study. Cancer. 1997;79(11):2087-2094.

16. Luo J, Guo RP, Lai EC, et al. Transarterial chemoembolization for unresectable hepatocellular carcinoma with portal vein tumor thrombosis: a prospective comparative study. Ann Surg Oncol. 2011;18(2):413-420.

17. Okusaka T, Okada S, Ishii H, et al. Prognosis of hepatocellular carcinoma patients with extrahepatic metastases. Hepatogastroenterology. 1997;44(13):251-257.

18. Jun $\mathrm{CH}$, Yoon $\mathrm{JH}$, Cho E, et al. Barcelona clinic liver cancer-stage $\mathrm{C}$ hepatocellular carcinoma: a novel approach to subclassification and treatment. Medicine. 2017;96(17):e6745.
19. Katagiri S, Yamamoto M. Multidisciplinary treatments for hepatocellular carcinoma with major portal vein tumor thrombus. Surg Today. 2014;44(2):219-226.

20. Huang W-K, Yang S-F, You L-N, et al. Transcatheter arterial chemoembolisation (TACE) plus S-1 for the treatment of BCLC stage B hepatocellular carcinoma refractory to TACE. Wspótczesna Onkologia. 2016;6(6):468-474.

21. Lencioni R, Llovet JM. Modified RECIST (mRECIST) assessment for hepatocellular carcinoma. Semin Liver Dis. 2010;30(1):52-60.

22. Shim JH, Lee HC, Kim SO, et al. Which response criteria best help predict survival of patients with hepatocellular carcinoma following chemoembolization? A validation study of old and new models. Radiology. 2012;262(2):708-718.

23. Uraki J, Yamakado K, Nakatsuka A, Takeda K. Transcatheter hepatic arterial chemoembolization for hepatocellular carcinoma invading the portal veins: therapeutic effects and prognostic factors. Eur J Radiol. 2004;51(1):12-18.

24. Nakao N, Uchida H, Kamino K, et al. Determination of the optimum dose level of lipiodol in transcatheter arterial embolization of primary hepatocellular carcinoma based on retrospective multivariate analysis. Cardiovasc Intervent Radiol. 1994;17(2):76-80.

25. Chung GE, Lee JH, Kim HY, et al. Transarterial chemoembolization can be safely performed in patients with hepatocellular carcinoma invading the main portal vein and may improve the overall survival. Radiology. 2011;258(2):627-634.

26. Song DS, Song MJ, Bae SH, et al. A comparative study between sorafenib and hepatic arterial infusion chemotherapy for advanced hepatocellular carcinoma with portal vein tumor thrombosis. J Gastroenterol. 2015;50(4):445-454.

27. Niu ZJ, Ma YL, Kang P, et al. Transarterial chemoembolization compared with conservative treatment for advanced hepatocellular carcinoma with portal vein tumor thrombus: using a new classification. Med Oncol. 2012;29(4):2992-2997.
Cancer Management and Research

\section{Publish your work in this journal}

Cancer Management and Research is an international, peer-reviewed open access journal focusing on cancer research and the optimal use of preventative and integrated treatment interventions to achieve improved outcomes, enhanced survival and quality of life for the cancer patient. The manuscript management system is completely online and includes

\section{Dovepress}

a very quick and fair peer-review system, which is all easy to use. Visit http://www.dovepress.com/testimonials.php to read real quotes from published authors. 